This item was submitted to Loughborough's Research Repository by the author.

Items in Figshare are protected by copyright, with all rights reserved, unless otherwise indicated.

\title{
Computational analysis of mechanical stress-strain interaction of a bioresorbable scaffold with blood vessel
}

PLEASE CITE THE PUBLISHED VERSION

http://dx.doi.org/10.1016/j.jbiomech.2016.05.035

PUBLISHER

(c) Elsevier

VERSION

AM (Accepted Manuscript)

\section{PUBLISHER STATEMENT}

This work is made available according to the conditions of the Creative Commons Attribution-NonCommercialNoDerivatives 4.0 International (CC BY-NC-ND 4.0) licence. Full details of this licence are available at: https://creativecommons.org/licenses/by-nc-nd/4.0/

\section{LICENCE}

CC BY-NC-ND 4.0

\section{REPOSITORY RECORD}

Schiavone, Alessandro, C. Abunassar, S. Hossainy, and Liguo Zhao. 2019. "Computational Analysis of Mechanical Stress-strain Interaction of a Bioresorbable Scaffold with Blood Vessel". figshare. https://hdl.handle.net/2134/21737. 


\title{
Computational Analysis of Mechanical Stress-Strain Interaction of a Bioresorbable Scaffold with Blood Vessel
}

\author{
A. Schiavone ${ }^{1}$, C. Abunassar ${ }^{2}$, S. Hossainy ${ }^{2}$, L.G. Zhao ${ }^{1 *}$, \\ ${ }^{1}$ Wolfson School of Mechanical, Electrical and Manufacturing Engineering, Loughborough \\ University, Epinal Way, Loughborough, LE11 3TU, UK \\ ${ }^{2}$ Abbott Vascular, 3200 Lakeside Drive, Santa Clara, CA 95054, USA
}

*Corresponding author; Email: L.Zhao@Lboro.ac.uk; Tel.: 0044-1509-227799; Fax: 0044-1509227648

\begin{abstract}
Crimping and deployment of bioresorbable polymeric scaffold, Absorb, were modelled using finite element method, in direct comparison with Co-Cr alloy drug eluting stent, Xience V. Absorb scaffold has an expansion rate lower than Xience V stent, with a less outer diameter achieved after balloon deflation. Due to the difference in design and material properties, Absorb also shows a higher recoiling than Xience $\mathrm{V}$, which suggests that additional post-dilatation is required to achieve effective treatment for patients with calcified plaques and stiff vessels. However, Absorb scaffold induces significantly lower stresses on the artery-plaque system, which can be clinically beneficial. Eccentric plaque causes complications to stent deployment, especially non-uniform vessel expansion. Also the stress levels in the media and adventitia layers are considerably higher for the plaque with high eccentricity, for which the choice of stents, in terms of materials and designs, will be of paramount importance. Our results imply that the benefits of Absorb scaffolds are amplified in these cases.
\end{abstract}

Keywords: Bioresorbable scaffold; Stent crimping; Stent deployment; Stresses; Eccentric plaque. 


\section{Introduction}

Development of next-generation polymeric bioresorbable scaffolds (BRSs), such as Igaki-Tamai, Abbott BVS and REVA stents (Waksman, 2006; Onuma and Serruys, 2011), are still in the infancy stage, with many unresolved issues regarding the material, design, biodegradation, biocompatibility, fabrication and in-vivo performance. One of the concerns for polymeric BRSs is their mechanical performance, especially their interaction with blood vessels during and post deployment. Finite element (FE) method has been particularly useful in understanding stent performance. However, the majority of FE analyses of stent deployment, including many of the latest ones, were focused on the mechanical behaviour of metallic stents, i.e., expansion, dogboning, recoiling, design and stent-artery interaction (e.g. Chua et al., 2003; Lally et al., 2005; Schiavone et al., 2014). There is extremely limited work devoted to modelling the deformation of bioresorbable polymeric stents, especially comparative studies against widely used metallic stents, which is important to evaluate the performance of these new-generation devices. We only came across two papers by Pauck and Reddy (2014) and Debusschere et al. (2015), respectively, who carried out computational analyses of expansion of PLLA stents (by an inflating balloon). Pauck and Reddy (2014) compared the mechanical performance of PLLA stents for three different geometries with varying material stiffness. The radial strength was largely dependent on material properties and stent design. Debusschere et al. (2015) focused on the development of an implicit finite element strategy, as opposed to an explicit one, to study the mechanical behaviour, such as stress distribution, recoiling and dogboning effects, of PLLA stents. However, both works ignored the diseased artery in their simulations, and further work is required to understand the mechanical behaviour of PLLA stents during the process of deployment inside diseased arteries.

In addition, a recent study by Liu et al. (2014) identified the complex pathological morphology of atherosclerotic blood vessels, such as vessel tortuosity, plaque asymmetry and varied severity of 
stenosis, using magnetic resonance imaging and computed tomography scan, which can play an important role in stent deployment. For instance, Wu et al. (2007) compared stent expansion in curved and straight arteries, and showed that artery curvature tended to increase the stress levels on both the plaque and the arterial layers. Gu et al. (2012) studied the arterial wall mechanics during stent deployment by considering an asymmetric plaque layer with an asymmetry ratio of 2:1 and an overall stenosis of $50 \%$. The results showed that, in addition to stent design, non-uniform plaque thickness due to its asymmetry significantly affects the stresses in the artery induced by stent deployment. An increase in plaque thickness or stiffness (e.g., plaque calcification) tended to reduce the stress concentration in the arterial layers. But work in this area is very scarce according to our literature search, and there is a serious lack of understanding about the effect of abnormal arterial factors on stent deployment and the consequent mechanical complications to blood vessels caused by stenting procedure.

In this paper, deployment of the Absorb (Abbott Vascular, USA) bioresorbable scaffold was simulated and compared with the metallic Xience V stent (MultiLink Vision platform design, Abbott Vascular, USA). In particular, the simulation accounted for the crimping process, which is an essential step for fixing the as-manufactured stent onto the balloon catheter and tends to generate high level residual stresses on the stent. A comparison has been made to assess the performances of the two stents, based on (1) the radial expansion characteristics of the arteryplaque system and (2) the stresses in both the stent and the blood vessel. To elucidate the effect of eccentric plaque on stent deployment, simulations were also made by considering plaque asymmetry, with a 9:1 asymmetric ratio, for both implants. 


\section{Description of the Model}

\subsection{Models for stents, plaque-artery and balloon}

Device geometries were created using ABAQUS CAE, based on the image of actual scaffolds in expanded shape (Figure 1). For both scaffolds, the expanded or initial outer diameter is measured to be $3 \mathrm{~mm}$ and the length is chosen to be $10 \mathrm{~mm}$. The strut thickness is $80 \mu \mathrm{m}$ and $150 \mu \mathrm{m}$ for Xience V and Absorb, respectively. The folded balloon has a main diameter of $1.25 \mathrm{~mm}$ and an overall length of $10 \mathrm{~mm}$, with both ends fully constrained.

The artery (refers to left anterior descending coronary artery here) has an inner diameter of $3 \mathrm{~mm}$ and a length of $40 \mathrm{~mm}$. The healthy wall thickness was chosen to be $1 \mathrm{~mm}$, and consists of three tissue layers, i.e., intima, media and adventitia, with a thickness of $0.27 \mathrm{~mm}, 0.35 \mathrm{~mm}$ and 0.38 $\mathrm{mm}$, respectively (Holzapfel et al., 2005). The plaque layer is located in the middle part of the artery with a total length of $10 \mathrm{~mm}$ and a stenosis of $50 \%$ (calculated as the ratio of the plaque thickness to the inner radius of a healthy artery). In the concentric case, the plaque was modelled with perfect symmetry, whilst in the eccentric case, the plaque is asymmetric with an edge ratio, defined as the ratio between the maximum and the minimum thickness, of 9:1 (Figure 2).

The stent was meshed using 8-node hexahedral elements with full integration and incompatible modes (C3D8I), which is suitable for modelling large bending deformation. All struts have four layers of elements through both the thickness and the width. The balloon was meshed using 4node shell elements with reduced integration (S4R). The artery-plaque system was meshed using 8-node hexahedral elements with reduced integration (C3D8R). There are four layers of elements through the thickness of each arterial layer and eight layers of elements through the thickness of the plaque. Figures 1 and 2 show the meshes created for the stent, balloon and artery-plaque (both eccentric and concentric cases). 


\subsection{Constitutive behaviour of materials}

The Xience V stent and the Absorb scaffold were both modelled with elastic-plastic properties and non-linear hardening, based on the tensile stress-strain curves of the materials. For Co-Cr L605, stress-strain curve and elastic properties can be referred to Schiavone et al. (2104). For PLLA, the stress-strain curve was created based on the average of uniaxial tensile test results (circumferential direction) in Pauck and Reddy (2014), from which Young's modulus (2.2 GPa), yield stress $(60 \mathrm{MPa})$ and strain hardening were extracted. Hardening was implemented in ABAQUS by considering the increase of yield stress with the plastic strain. The hypocellular plaque was modelled as an isotropic hyperelastic material using the Ogden strain energy potential, with parameters given in Zahedmanesh and Lally (2009). While the balloon was modelled as linear elastic, with a density of $1.1 \times 10^{6} \mathrm{~kg} / \mathrm{mm}^{3}$, Young's modulus of $900 \mathrm{MPa}$ and Poisson's ratio of 0.3 (Gervaso et al., 2008). The HGO model (Holzapfel et al., 2000) was used to describe the anisotropic constitutive behaviour of arterial layers (reinforced with two families of fibre). The HGO model parameters for the three layers were calibrated against the experimental data in Holzapfel et al. (2005) and given in Schiavone and Zhao (2016).

\subsection{Modelling of stent crimping}

To simulate the crimping of the stent, a series of 12 rigid planes were created around the scaffold in a cylindrical pattern. A displacement boundary condition was enforced to the planes in the radial direction with a magnitude linearly increasing to $1 \mathrm{~mm}$. Spring back $(\sim 0.2 \mathrm{~mm})$ of the scaffold after crimping resulted in a final released outer diameter of $1.5 \mathrm{~mm}$, which fits inside the diseased artery. The Abaqus/Explicit solver was used for this simulation, with a step time of $0.1 \mathrm{~s}$. Hard contact with a friction coefficient of 0.8 was applied between the rigid planes and the outer surface of the stent. At the end of the crimping, one additional step (0.1 s) was implemented to allow the spring back of the stent from the fully crimped state. 


\subsection{Modelling of stent deployment}

The expansion procedure was simulated using two steps, namely inflation and deflation. During the inflation step a pressure linearly increasing to a maximum of $1.2 \mathrm{MPa}$ was applied, whilst in the deflation step, the balloon pressure was brought linearly to zero. In our simulation, zeropressure also indicates zero stress state, as the intrinsic residual stresses in the artery system was not considered. However, the residual stresses generated during the crimping process were accounted for in all simulations. Simulations were performed using Abaqus/Explicit solver with controlled time increments (on the order of $10^{-8} \mathrm{~s}$ ). The interaction between the stent, the balloon and the artery/plaque was modelled using hard contact formulation. A friction coefficient of 0.25 (Ju et al., 2008) was assigned for the contact surfaces to avoid sliding of the stent in the longitudinal direction. Outer diameter change was monitored at 5 even points in the middle ring of the stent. The overall expansion of the stent was then calculated as the mean of the values obtained at those 5 points. Outer diameter change at both ends of the stent was also monitored at 5 even points along the circumferential ring to calculate the dogboning effect. The recoiling was calculated as $\left(d_{0}-d_{1}\right) / d_{0}$, where $d_{0}$ and $d_{1}$ are the mean outer diameters for the middle ring of the stent at the peak of inflating pressure and in the end of balloon deflation, respectively. In the case of eccentric plaque, the expansion of the artery and the recoiling/dogboning were monitored at the top and bottom locations of the lumen, corresponding to the points where the plaque has the maximum and minimum thickness and also the outer diameter of the stent. The interaction between the blood flow and blood vessel was not considered in this paper and will be explored in future work.

\section{Results and Discussion}

\subsection{Residual stresses due to crimping}

The maximum von Mises stress after crimping were $750 \mathrm{MPa}$ for the Xience V stent and $97 \mathrm{MPa}$ for the Absorb scaffold (Figure 3a). They were found at the inner and outer sides of the U-bends 
(crests) for both stents. After spring back, the residual stresses reduced to $706 \mathrm{MPa}$ and $85 \mathrm{MPa}$ for the Xience V stent and Absorb scaffold, respectively (Figure 3b), and the stents settled at an outer diameter of around $1.5 \mathrm{~mm}$. Crimping of the stent induced high levels of stress in the stent struts, which are well beyond the yield stress level. This indicates that the stent structure has accumulated significant plastic strain during crimping before the inflation process begins. These residual stresses are believed to affect the nature of stent deformation during the subsequent expansion process (Schiavone and Zhao, 2016).

\subsection{Stent deployment}

As shown in Figure 4a, the Absorb scaffold expanded with a slower rate when compared to Xience V stent. However, the outer diameter at the maximum inflating pressure was very similar for both cases and computed to be 2.66 for Xience $\mathrm{V}$ and $2.67 \mathrm{~mm}$ for Absorb. The recoiling for the Xience V (11\%) (Figure 4b) was lower than that for the Absorb scaffold (17\%), leading to a final outer diameter of $2.37 \mathrm{~mm}$ for the Xience V stent and $2.22 \mathrm{~mm}$ for the Absorb scaffold. In addition, the Xience V stent also experienced less dogboning behaviour (24\%) than the Absorb scaffold $(33 \%)$.

After balloon deflation, the maximum von Mises stress occurred in the inner corners of the Ubends with a magnitude of $935 \mathrm{MPa}$ and $97 \mathrm{MPa}$ for the Xience V and Absorb, respectively. Figure 6 shows the maximum principal stress in the artery-plaque system at the end of balloon deflation. The maximum stress was located on the inner surface of the plaque, with strong concentration at both ends of the stenosis due to the dogboning effect (Figure 6a). The maximum stress on the plaque was 1.43 MPa for the Xience V stent and only 0.75 MPa for Absorb scaffold (reduced by almost 50\%). Similar behaviour was also observed for individual vessel layer (Figure $6 \mathrm{~b}$ to $6 \mathrm{~d}$ ). 
For both stents, the target outer diameter of $3 \mathrm{~mm}$ is not achieved in a single simulated inflation. This is due to the expansion saturation of the artery, mainly governed by the high stiffness of the intima layer developed at the late stage of stretching. Increased recoiling effect is also found for the Absorb scaffold due to the weaker strength of PLLA compared to that of Co-Cr alloy. This finding suggests that additional balloon post-dilation is likely required for successful deployment of bioresorbable PLLA stents in patients with high arterial stiffness and plaque calcification. Once deployed, it is noticed that Absorb scaffold induces significantly lower stresses on the artery-plaque system than Xience V stent, along with lower contact pressures. This is due to reduced system expansion or vessel stretch for Absorb. In addition, there is much less property mismatch between PLLA and artery system, which contributed to the lower stresses in the tissue layers. Furthermore, the vessel-stent contacting surface area for Absorb is greater than that for Xience V, which plays a role in distributing stress more evenly and serves as another mechanism of lower arterial stress.

Clinically, the major drawback associated with stent implantation is in-stent restenosis (ISR), i.e., re-narrowing of the cross section of artery. ISR is largely a result of neointima formation alone which is composed principally of proliferating smooth muscle cell and accumulated extracellular matrix (Lowe et al., 2002). Stents that induce higher non-physiologic stresses provoke a more aggressive pathobiological response of the artery wall, resulting in a higher degree of neointimal hyperplasia (Timmins et al., 2011). Absorb scaffold is clinically beneficial due to the lower stresses generated in the vessel layers (i.e., lower risks of restenosis, chest pain and arterial dissection or perforation). But it should be pointed out that there is also a sacrifice of smaller lumen diameter for Absorb.

\subsection{Effect of lesion eccentricity}

Stent deployment within a vessel with eccentric plaque led to an unequal radial expansion of the stent. As shown in Figure 7a, the radial expansion of the Absorb scaffold was considerably higher 
on the side with the minimum thickness of plaque, compared to the side with the maximum plaque thickness. Radial expansion is defined from the centre of the original lesion, which was treated as fixed. At the side with the thickest plaque, stent needs to push away both the plaque and the artery to make any expansion. Consequently, it has a lower radial expansion than the side with the thinnest plaque, even though the plaque is more compliant than the artery. The lumen radius after balloon deflation settled at $1.13 \mathrm{~mm}$ and $1.38 \mathrm{~mm}$ for the sides with the thickest and the thinnest plaque, respectively. However, the average expansion was found similar, regardless of concentric or eccentric plaque layer, with a final outer diameter of $2.40 \mathrm{~mm}$ for the Xience V stent and 2.21 $\mathrm{mm}$ for the Absorb scaffold. The cross section of the deployed Absorb scaffold showed a more eccentric shape than that of the Xience V stent. This is consistent with observations in clinical practice which confirmed that Absorb scaffolds tended to result in a more eccentric final deployment shape as compared to the Xience V stents.

Plaque asymmetry also caused the non-uniform development of dogboning and recoiling (Figure $7 b)$. For Absorb, the dogboning was around $75 \%$ when calculated for the side with the maximum thickness and reduced dramatically (almost zero) when calculated for the side with the minimum thickness (Figure 7b). Such non-uniformity was less pronounced for the Xience V stent. As expected, stresses on the stents were not affected by the plaque asymmetry, and both stents experienced similar levels of stress when compared to those obtained for symmetric plaque model.

\subsection{Stress profiles in the arterial layers}

For the eccentric plaque model, the stress distribution in the plaque and the vessel layers is largely non-uniform along the circumferential direction. For both scaffolds, the stress on the plaque was considerably higher in the regions with the maximum plaque thickness (Figure 8a), with a value of 2.58 $\mathrm{MPa}$ for the Xience V stent and 2.66 MPa for the Absorb scaffold. In the arterial layers, the stresses are found highly concentrated in the regions covered with thin plaque layer, which is the 
case for both Absorb scaffold and Xience V stent. Specifically, in the intima layer (Figure 8b), the stress has a maximum value of $2.15 \mathrm{MPa}$ for Xience V and 2.26 MPa for Absorb. These stresses are considerably higher than those, generally below $0.33 \mathrm{MPa}$ (Figure $8 \mathrm{c}$ and $8 \mathrm{~d}$ ), in the media and adventitia layers. The plaque and intima have higher stress level for the Absorb, indicating the higher risk of rupture of these tissue layers. This is more associated with the thicker struts of Absorb, and plaque rupture could be beneficial for stent deployment.

Figure 9 compares the volume averaged (or thickness averaged) maximum principal stresses as a function of longitudinal position in the media and adventitia layers for the concentric and eccentric plaque models. Medial stress affects smooth muscle cells and fibroblasts, and plays a key part in both restenosis phenomena and healing of the implant site. Stresses in the adventitia layer are of interest, since this is known to be more innervated and high stresses in this vascular layer could relate to increased levels of chest pain post-stenting. In truth, the damage of the internal elastic lamina (border between intima and media) or external elastic lamina (border between media and adventitia) has been strongly correlated with neointimal growth. This deep injury concept points to media and adventitia (Gunn et al., 2002). The expansion of a stent within an eccentric artery induced considerably higher stresses in these two vessel layers. Peak stress levels were normally located in the centre region of diseased arteries, which was in strong contact with the stent. The high stress peaked in the arterial layers can facilitate a cascade of undesired biological responses, including cellular injury, denudation of the endothelium, and the proliferation of smooth muscle vessel cells and increase the chances of restenosis. From ABSORB Cohort B (Absorb BVS 1.1) and SPIRIT FIRST (Xience V Multi-Link Vision) trials, García-García et al. (2014) calculated the total plaque area changes by intravascular ultrasound technique. Comparison at 6-month followup showed that both devices induced an increase in the total plaque area. However, a larger increase occurred with Xience V stents as compared to Absorb scaffolds. In the long term (from year 1 to 3), coronary polymeric scaffolds even allowed plaque regression. Interestingly, when 
looking at the lumen changes between 6 months and 2 years, Absorb always showed expansive remodelling while Xience V showed restrictive remodelling at all the time points (García-García et al., 2014). These clinical observations were supported by the low stress/strain levels induced in the vessel layers when treated with Absorb scaffold. The low level of vessel deformation also implied that Absorb scaffold had better conformability than Xience V stent, allowing the restoration of the coronary geometry (vessel curvature and angulation).

\section{Conclusions}

Absorb scaffold induces significantly lower stresses on the artery-plaque system than Xience $\mathrm{V}$ stent, along with lower contact pressures. This is clinically beneficial and associated with the lower angina rates observed with Absorb when compared to Xience V. The benefits of Absorb scaffolds are amplified in patients with eccentric plaque, as the higher stress levels encountered for plaques with high eccentricity can be largely mediated by the polymer scaffold. Absorb scaffold gives lower expansion and higher recoiling, which, however, can be overcome by additional post-dilatation.

5. Conflict of interest: The authors declare no conflict of interest.

\section{References}

Chua, S.D., Mac Donald, B., Hashmi, M., 2003. Finite element simulation of stent and balloon interaction. Journal of Materials Processing Technology 143, 591-597.

Debusschere, N., Segers P., Dubruel P., Verhegghe B., De Beule M., 2015. A finite element strategy to investigate the free expansion behaviour of a biodegradable polymeric stent. Journal of Biomechanics 48, 2012-2018.

Gervaso, F., Capelli, C., Petrini, L., Lattanzio, S., Di Virgilio, L., Migliavacca, F., 2008. On the effects of different strategies in modelling balloon-expandable stenting by means of finite element method. Journal of Biomechanics 41, 1206-1212. 
Gu, L., Zhao, S., Froemming, S.R., 2012. Arterial wall mechanics and clinical implications after coronary stenting: comparisons of three stent designs. International Journal of Applied Mechanics 4, 1250013-1250027.

García-García, H.M., Serruys, P.W., Campos, C.M., Onuma Y., 2014. Differential impact of five coronary devices on plaque size: Insights from the ABSORB and SPIRIT trials. International Journal of Cardiology 175, 441-445.

Gunn, J., Arnold, N., Chan, K.H., Shepherd, L., Cumberland, D.C., Crossman, D.C., 2002. Coronary artery stretch versus deep injury in the development of in-stent neointima. Heart $88,401-405$.

Holzapfel, G.A., Gasser T.C., Ogden R.W., 2000. A new constitutive framework for arterial wall mechanics and a comparative study of material models. Journal of Elasticity and the Physical Science of Solids 61, 1-48.

Holzapfel, G.A., Sommer G., Gasser C.T., Regitnig, P., 2005. Determination of layer-specific mechanical properties of human coronary arteries with nonatherosclerotic intimal thickening and related constitutive modeling. American Journal of Physiology-Heart and Circulatory Physiology 289, H2048-H2058.

Ju, F., Xia, Z. Sasaki, K., 2008. On the finite element modelling of balloon-expandable stents. Journal of the Mechanical Behavior of Biomedical Materials 1, 86-95.

Lally, C., Dolan, F., Prendergast, P., 2005. Cardiovascular stent design and vessel stresses: a finite element analysis. Journal of Biomechanics 38, 1574-1581.

Liu, J., Jia, X.-J., Wang, Y.-J., Zhang, M., Zhang, T., Zhou, H.-D., 2014. Digital subtraction angiography imaging characteristics of patients with extra-intracranial atherosclerosis and its relationship to stroke. Cell Biochemistry and Biophysics 69, 599-604.

Lowe, H.C., Oesterle, S.N., Khachigian, L.M., 2002. Coronary in-stent restenosis: current status and future strategies. Journal of the American College of Cardiology 39, 183-193. 
Onuma, Y., Serruys, P.W., 2011. Bioresorbable scaffold: the advent of a new era in percutaneous coronary and peripheral revascularization? Circulation 123, 779-797.

Pauck, R.G., Reddy, B.D. 2014. Computational analysis of the radial mechanical performance of pllacoronary artery stents. Medical Engineering \& Physics 37, 7-12.

Schiavone, A., Zhao, L.G., Abdel-Wahab, A.A., 2014. Effects of material, coating, design and plaque composition on stent deployment inside a stenotic artery - finite element simulation. Materials Science and Engineering C 42, 479-488.

Schiavone, A., Zhao, L.G., 2016. A computational study of stent performance by considering vessel anisotropy and residual stresses. Materials Science and Engineering C, in press.

Timmins, L.H., Miller, M.W., Clubb Jr., F.J., Moore Jr., J.E., 2011. Increased artery wall stress post-stenting leads to greater intimal thickening. Laboratory Investigation 91, 955-967.

Waksman, R.., 2006. Biodegradable stents: they do their job and disappear. The Journal of Invasive Cardiology 18, 70-74.

Wu, W., Wang, W.-Q., Yang, D.-Z., Qui, M., 2007. Stent expansion in curved vessel and their interactions: A finite element analysis. Journal of Biomechanics 40, 2580-2585.

Zahedmanesh, H., Lally, C., 2009. Determination of the influence of stent strut thickness using the finite element method: implications for vascular injury and in-stent restenosis. Medical \& Biological Engineering \& Computing 47, 385-393. 
(a)

(b)
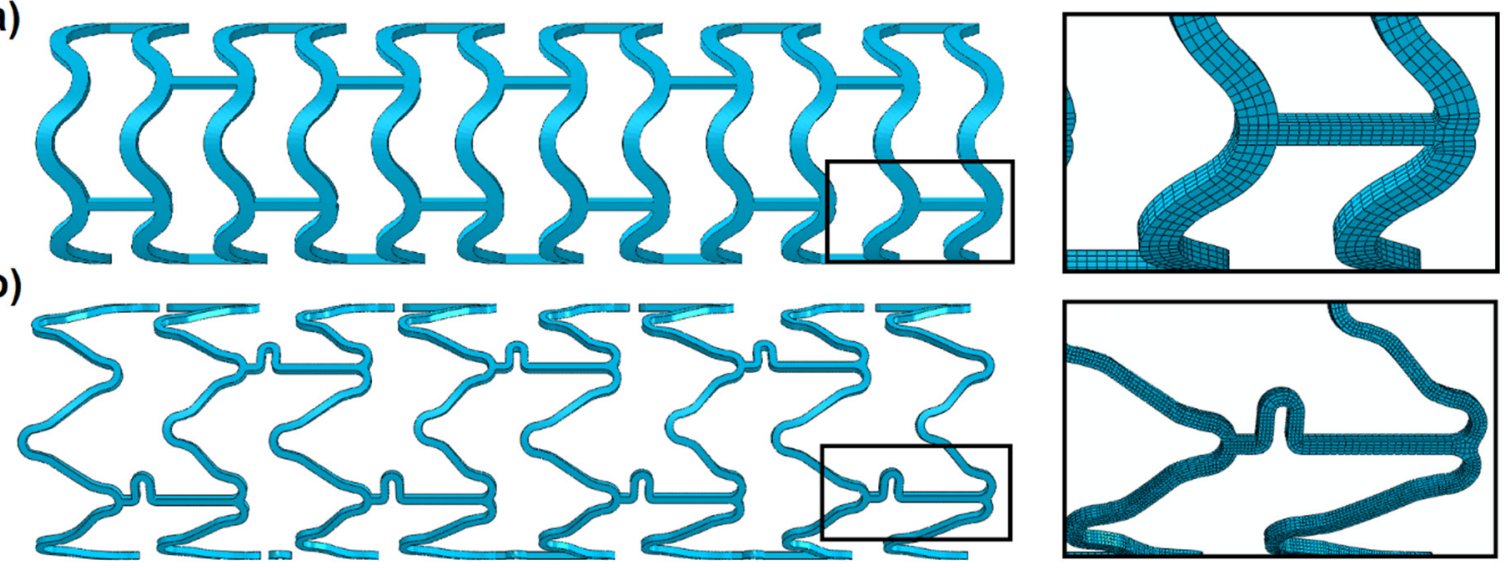

Figure 1, Expanded stent geometry and FE mesh for (a) Absorb scaffold and (b) Xience V stent. 
(a)

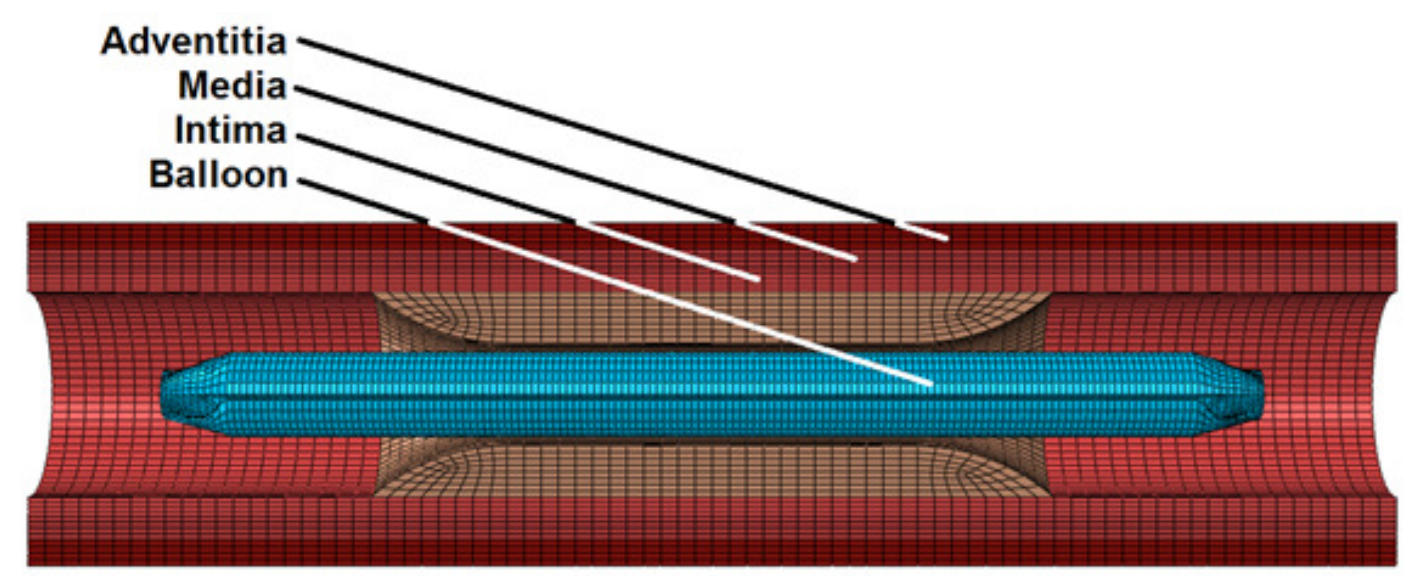

(b)

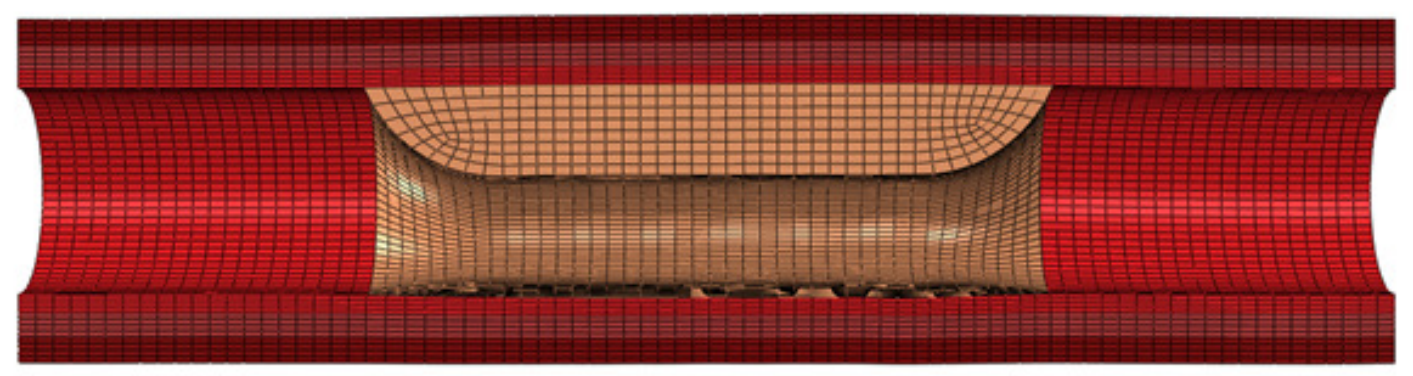

Figure 2, Geometry and mesh for artery with (a) concentric and (b) eccentric stenosis. 
(a)

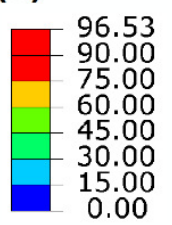

(b)

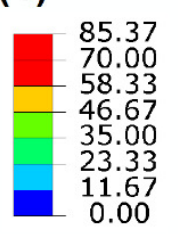

Absorb
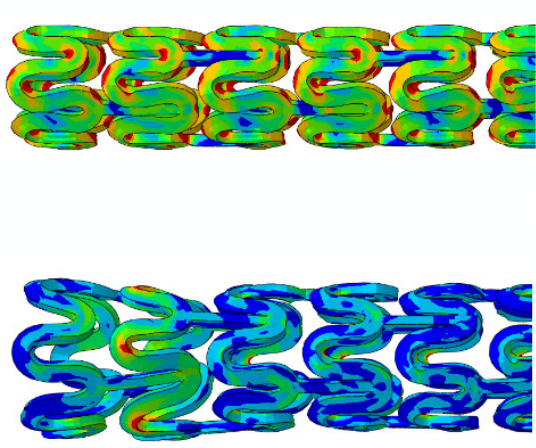

Xience $\mathrm{V}$
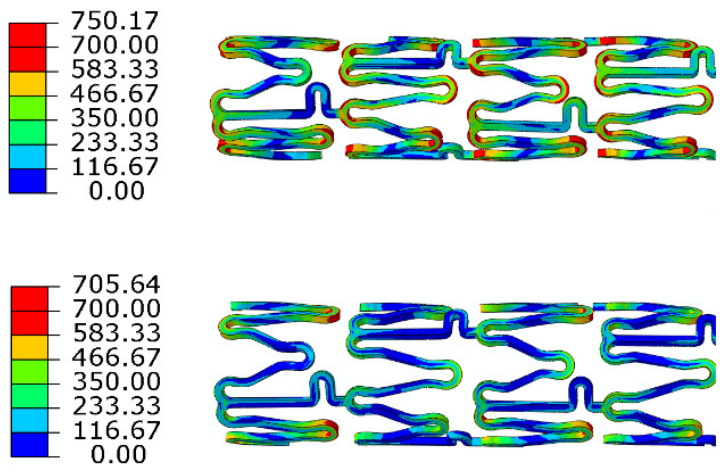

Figure 3, The von Mises stress (MPa) contour plot for the Absorb scaffold (left) and Xience V stent (right) in (a) fully crimped configuration and (b) after spring back. 
(a)
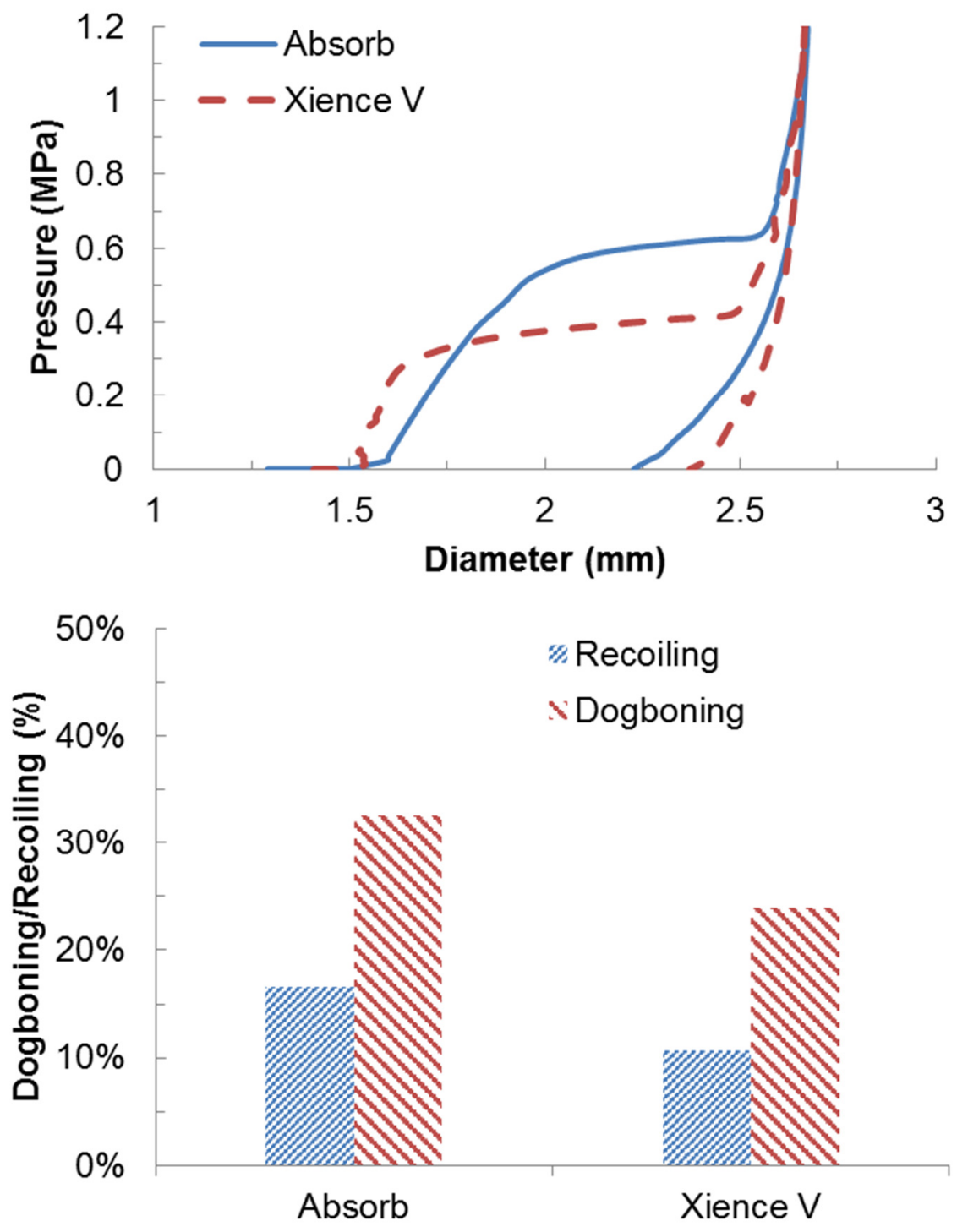

(b)

Figure 4, (a) Stent outer diameter change against pressure and (b) recoiling and dogboning effects for Absorb scaffold and Xience V stent in concentric lesion. 
(a)

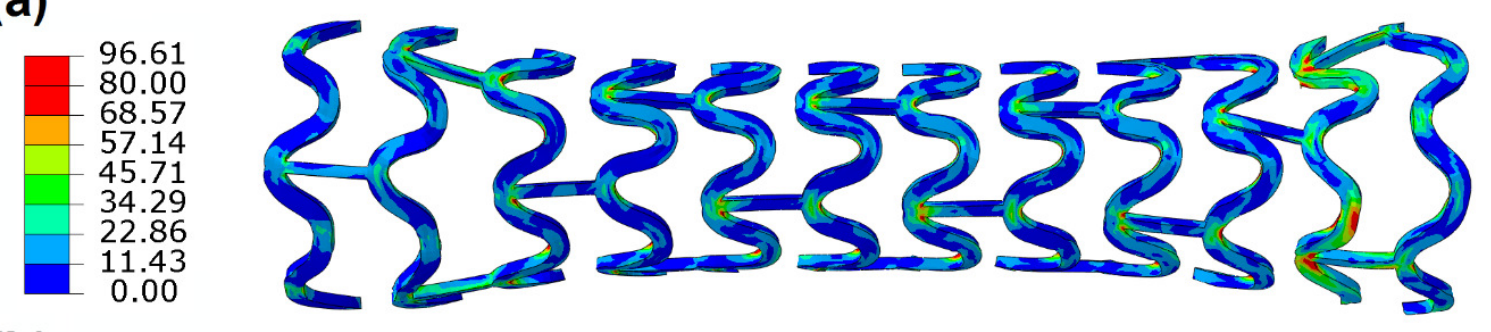

(b)

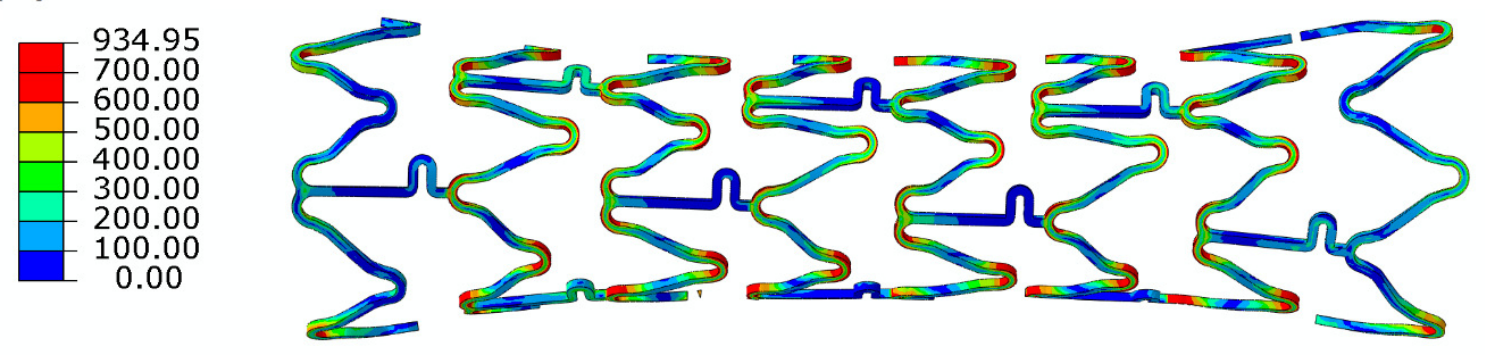

Figure 5, Contour plot of the von Mises stress (MPa) on (a) Absorb scaffold and (b) Xience V stent after deployment in concentric lesion. 
(a)

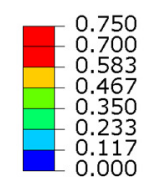

(b)
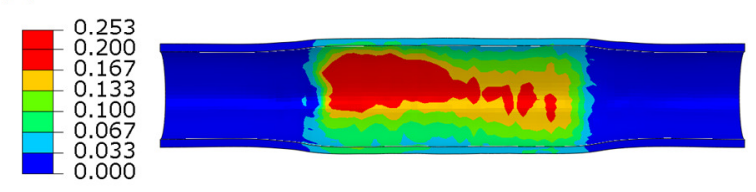

(c)
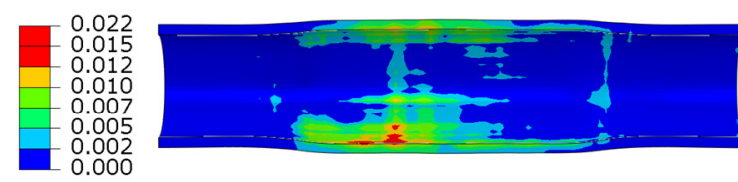

(d)
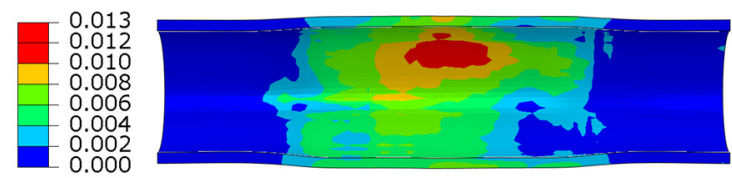

Xience V
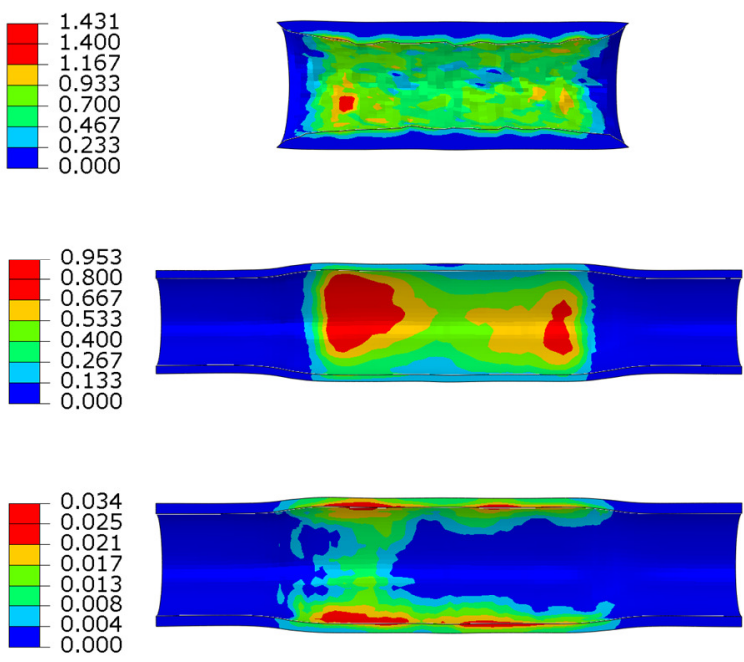

Figure 6, Contour plot of the maximum principal stress $(\mathrm{MPa})$ on the (a) plaque, (b) intima layer, (c) media layer and (d) adventitia layer, after deployment of Absorb scaffold and Xience V stent in concentric lesion. 

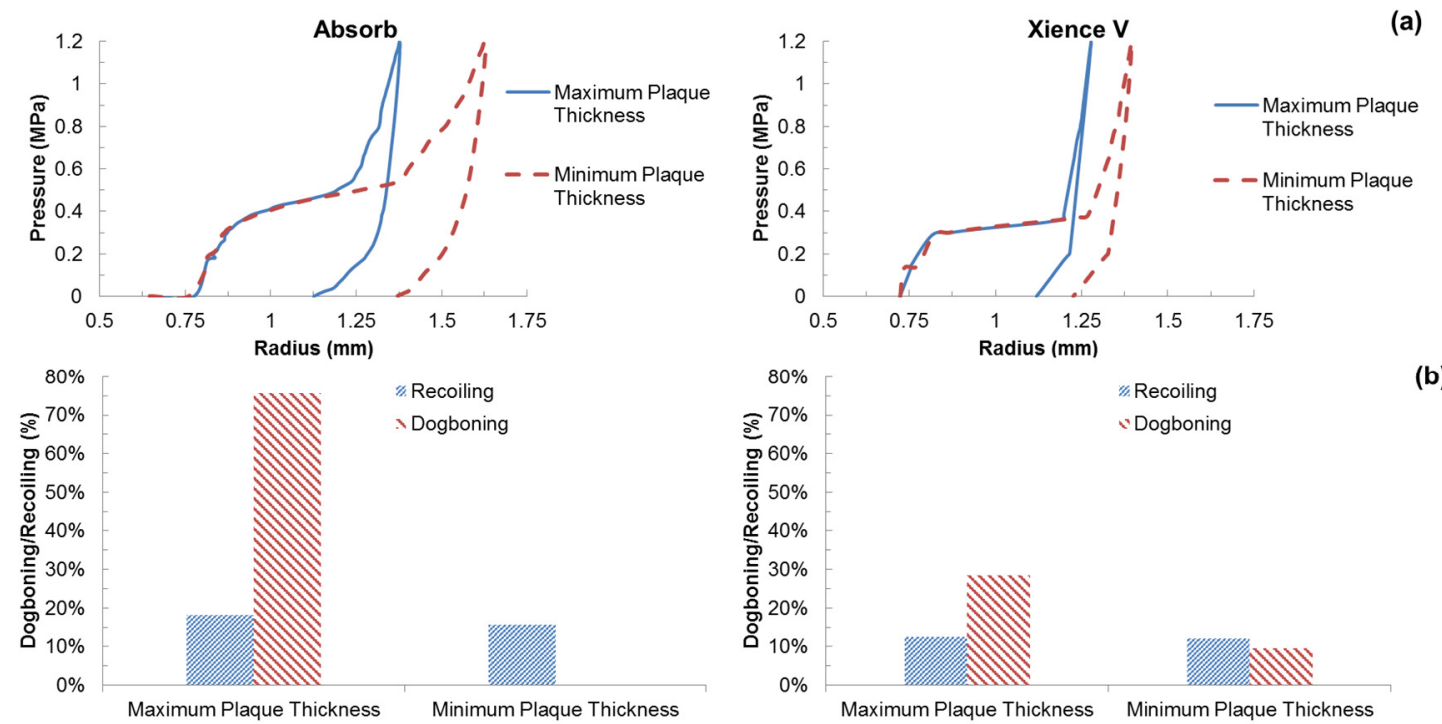

Figure 7, Radial expansion against balloon pressure for Absorb scaffold and Xience V stent at the sides with maximum and minimum thickness of plaque. 
(a)

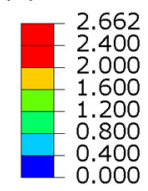

(b)
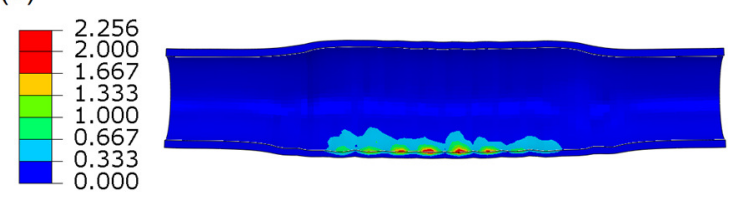

(c)
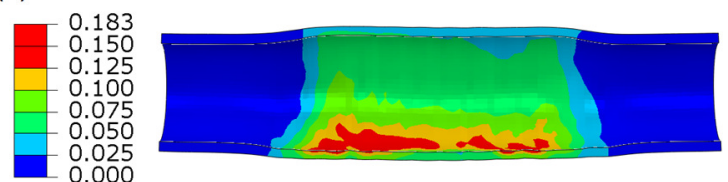

(d)
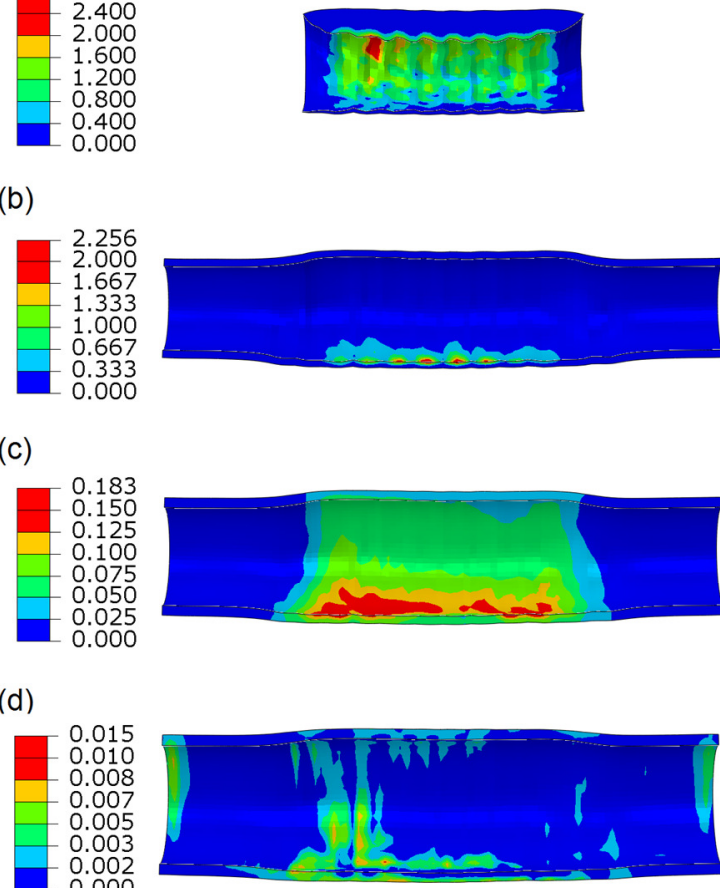

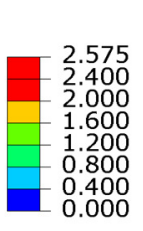

Xience $\mathrm{V}$
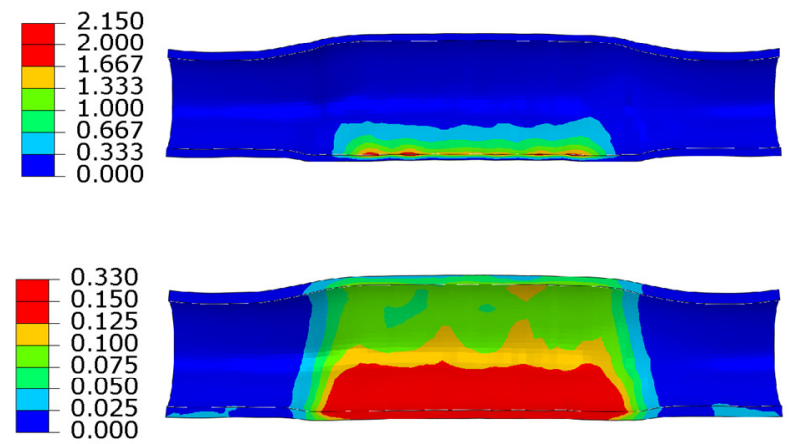

0.000

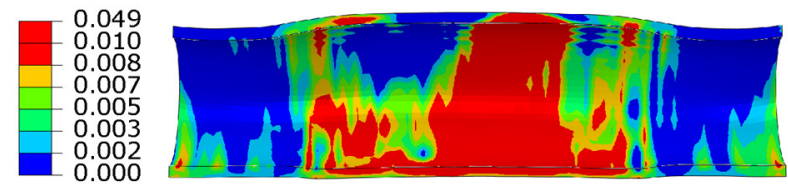

Figure 8, Contour plot of the maximum principal stress (MPa) on the (a) plaque, (b) intima layer, (c) media layer and (d) adventitia layer, after deployment of Absorb scaffold and Xience V stent in eccentric lesion. 
Concentric Lesion
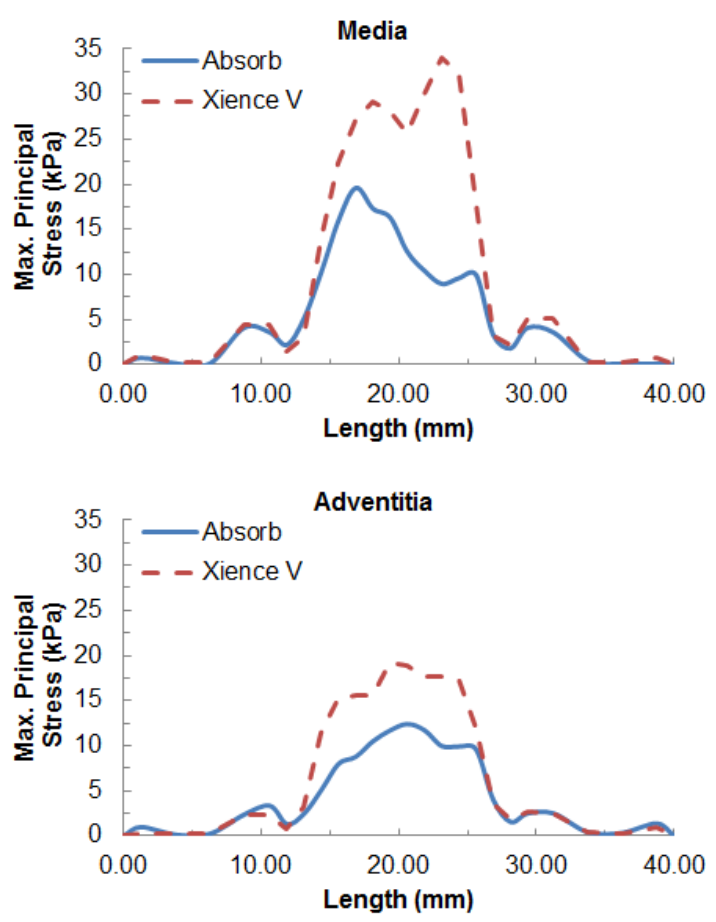

Eccentric Lesion
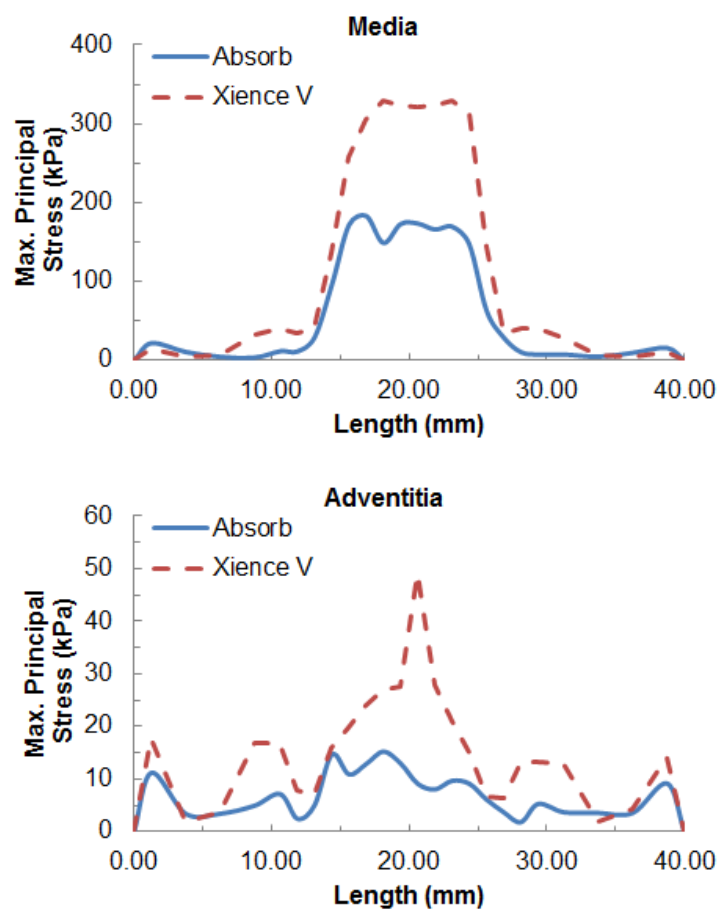

Figure 9, Maximum principal stress in the media and adventitia arterial layers plotted as a function of longitudinal position in both concentric vs eccentric lesions, with stents/scaffolds located approximately between $\mathrm{x}=15 \mathrm{~mm}$ and $\mathrm{x}=25 \mathrm{~mm}$. 